\title{
Computer simulation of flat geogrids based on Discrete Element Method (DEM)
}

\author{
Andrei Moshenzhal ${ }^{1, *}$ \\ ${ }^{1}$ LLC MIAKOM SPb, Saint-Petersburg, Russia
}

\begin{abstract}
The article presents the way of modelling flat geogrids using the Discrete Element Method (DEM). Simulation of geogrids is performed on the base of EDEM ${ }^{\circledR}$ software produced by DEM Solutions Ltd. The HertzMindlin model with bonds is used as a model of particles interaction. The presented method of geogrid simulation using EDEM ${ }^{\circledR}$ software has some peculiarities in the formation of material structure. We compared two types of geogrids with the same characteristics formed out of a variety of balls with different radii. This article provides the simulation results of geogrid testing by calculating the force causing $2 \%$ and $5 \%$ tension (elongation) of the material.
\end{abstract}

\section{Introduction}

The construction sector always paid a great attention to improving the safety and reliability of the constructed projects, including transport infrastructure facilities. Constant increase in the rate of construction activities preserving the reliability and safety of the entire building has led to the development of many engineering solutions to achieve this. One of the most common solutions is the use of geosynthetic materials. As practice shows, the reinforcing layers left positive recommendations for more than half a century of successful operation in various structures. But, unfortunately, at present, the interaction of geosynthetic material with a reinforced loose soil is not directly accounted in the calculation process. It is accounted by introduction of additional coefficients influencing on the change of strength and deformation parameters of the reinforced soil studied at the micro level. The latter is connected with the assumptions and provisions of the continuum mechanics, as well as with the used singularities of the mathematical apparatus, which do not allow reasonably estimating the collaboration of geogrids and soil particles.

It is known that there are so called bonds which occur in the loose soil due to stresses and through which normal and tangential stresses are transferred [1]. Obviously, the presence of geogrids in the soil leads to the change in number and nature of the contacts, through which the stress is to be transferred [2], i.e. the soil reinforcement will influence on the nature of stress distribution. Present studies of this issue at the micro level include methods considering the loose soil as the soil, which consists of individual particles (grains). One of these methods is the Discrete Element Method (DEM) represented by various software products, for example, EDEM ${ }^{\circledR}$ produced by DEM Solutions Ltd [3]. This method allows saving

\footnotetext{
* Corresponding author: a.moshenzhal@gmail.com
} 
considerable funds and time for field tests. All this including the capabilities of modern computer technologies make it possible to effectively investigate the actual interaction between particles and with geogrids in a loose soil. Thus, the general problem on the influence of reinforcing geogrids on the loose soil can be divided into three separate tasks:

1. Develop a computer model (DEM) of the flat geogrid and determine its strength properties according to applicable methods;

2. Determine model's parameters (DEM) and compare its behavior under stress with field tests of the similar soil;

3. Combine geogrid and soil models into one and compare with field tests of the reinforced soil.

This article deals with the method provided in the $1^{\text {st }}$ task above, using EDEM $®$ software.

\section{Parameters of the Hertz-Mindlin bonded model}

DEM provides a variety of models that allow to determine the parameters of particles interaction in the soil. Here in the frame of this study we took the Hertz-Mindlin bonded model. The interaction between particles is shown in Fig.1.

The model includes the following mathematical functions:

$$
\begin{aligned}
& \delta \mathrm{F}_{\mathrm{n}}=-\mathrm{v}_{\mathrm{n}} \mathrm{S}_{\mathrm{n}} \mathrm{A} \delta \mathrm{t} \\
& \delta \mathrm{F}_{\mathrm{t}}=-\mathrm{v}_{\mathrm{t}} \mathrm{S}_{\mathrm{t}} \mathrm{A} \delta \mathrm{t} \\
& \delta \mathrm{M}_{\mathrm{n}}=-\omega_{\mathrm{n}} \mathrm{S}_{\mathrm{n}} \mathrm{J} \delta \mathrm{t}, \\
& \delta \mathrm{M}_{\mathrm{t}}=-\omega_{\mathrm{t}} \mathrm{S}_{\mathrm{t}} \frac{\mathrm{J}}{2} \delta \mathrm{t}
\end{aligned}
$$

where:

$$
\begin{aligned}
& \mathrm{A}=\pi \mathrm{R}_{\mathrm{B}}^{2} \\
& \mathrm{~J}=\frac{1}{2} \pi \mathrm{R}_{\mathrm{B}}^{4}
\end{aligned}
$$

where $R_{B}$ - bond radius, $m ; S_{n}, S_{t}$ - normal and tangential stiffness of the bond, $N / m^{3} ; \delta t$ - time interval (step-out), $s ; v_{n}, v_{t}$ - normal and tangential velocity of particles, $\mathrm{m} / \mathrm{s} ; \omega_{\mathrm{n}}, \omega_{\mathrm{t}}$ - normal and tangential angular velocity of particles, rad/s; A - cross-section area of the bond, $\mathrm{m}^{2} ; \mathrm{J}$ - moment of inertia, $\mathrm{N}^{*} \mathrm{~m}^{2}$.

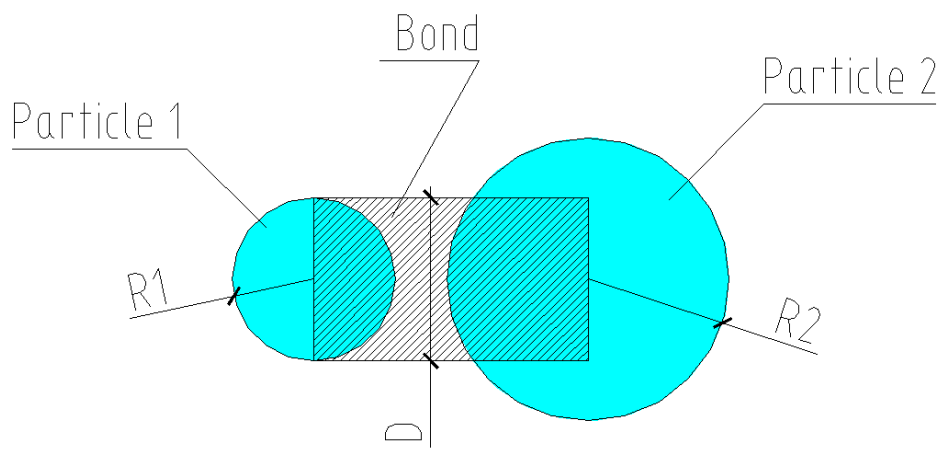

Fig.1. Single bond between two particles: R1, 2 - radius of the first and the second particle; D diameter of the bond between particles. 
Parameters $F_{n}, F_{t}$ are normal and tangential forces of the bonds between particles, $\mathrm{H}$, as well as $M_{n}, M_{t}$ - torsion moments, $N^{*} m$. Besides, the determination of those parameters may be interesting in the context of geogrid modelling with a respective tensile (or tear) strength. Here, as previously noted, it is interesting to obtain geogrid models, which behavior under stress at $2 \%$ and $5 \%$ tension [4] will correspond to the behavior of the real sample.

Laboratory tests of flat geogrids, as a rule, are performed without torsion and shear in the plane perpendicular to tension direction, therefore we use $R_{B}, S_{n}, R_{p}$ as the main HertzMindlin model parameters. Where $\mathrm{R}_{\mathrm{p}}$ - radius of particles forming the geogrid.

\section{Geogrid simulation}

The Hertz-Mindlin bonded model helps the researcher to connect an unlimited number of particles with each other by bonds, which have certain characteristics. Therefore, at first it is important to correctly set the geometric parameters of the material, which is planned to be formed from these particles, i.e. particles should fully define the shape of the model.

In $[5,6]$ works geogrid models have been made from small particles connected with each other by bonds having its certain characteristics. Unfortunately, the process of particles forming-up in a certain sequence is not described in those studies. But still it is important, since prior to bond connections between particles, those particles should be located in a certain way in relation to each other. Otherwise, the bonds can be destroyed in the process of geogrid formation or create an additional pre-stressing, having a possible negative impact the results of simulation. Thus, this article pays special attention to the issues concerning the formation of geogrid models.

Flat biaxially oriented geogrids are made of various materials using different technologies. This article describes studies, which developed two models of flat geogrids with characteristics similar to those made of polypropylene (

Fig. 2). The first model consists of particles with radius of $R_{P}^{1}=0.002475 \mathrm{M}$, and the second one - of particles with radius $\mathrm{R}_{\mathrm{P}}^{2}=0.000625 \mathrm{M}$. In both cases the geogrid thickness is $4.95 \mathrm{~mm}$.

Method in this article allows performing simulation of flat geogrids made of different materials, for example, polyester. The process of computer simulation complies with the requirements according to [7] of existing laboratory tests. We took a geogrid model of $200 \times 160 \mathrm{~mm}$ as a sample of the material, which was subjected to further tests. The parameters of the natural geogrid sample are shown in Table 1.

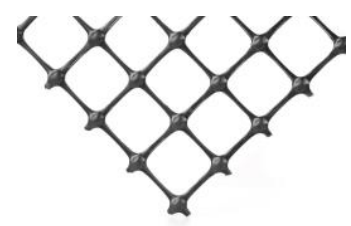

Fig. 2. General view of the polypropylene geogrid.

Table 1. The parameters of the sample for flat geogrid.

\begin{tabular}{|l|c|c|}
\hline Parameter & Unit & Value \\
\hline Maximum strength & $\mathrm{kN} / \mathrm{m}$ & 20 \\
\hline Tensile force $2 \%$ & $\mathrm{kN} / \mathrm{m}$ & 7 \\
\hline Tensile force $5 \%$ & $\mathrm{kN} / \mathrm{m}$ & 14 \\
\hline Grid (cell) size & $\mathrm{mm}$ & 40 \\
\hline
\end{tabular}


As previously said, simulation was performed using EDEM $^{\circledR}$ software, which allows importing sub-models made in other software. For the formation of a geogrid model it is necessary to locate particles in a certain sequence in relation to each other. Thus, we developed such model forms, in which geogrid models were generated (Fig. 3) in EDEM ${ }^{\circledR}$ environment. Form a) Fig. 3 has special edges allowing to fix the position of particles with radius $R_{P}^{1}=0.002475 \mathrm{M}$ in a certain sequence. Form b) has no vertical edges, thus allowing particles to interact and take the most suitable position without any pre-stressed bonds in the process of sample formation of the required geometry.

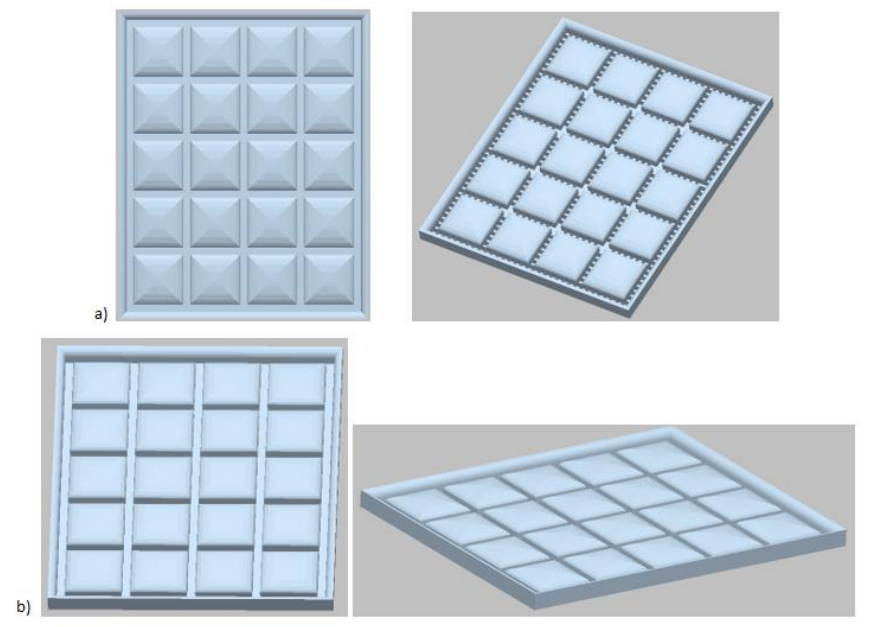

Fig. 3. General view of the model for geogrid simulation: a) - for the first model; b) - for the second model.

After particles have been placed in those forms, they were connected by bonds characterized by parameters of the Hertz-Mindlin bonded model. Therefore, we obtained both geogrid models. General view of those models are shown in Fig. 4.

a)

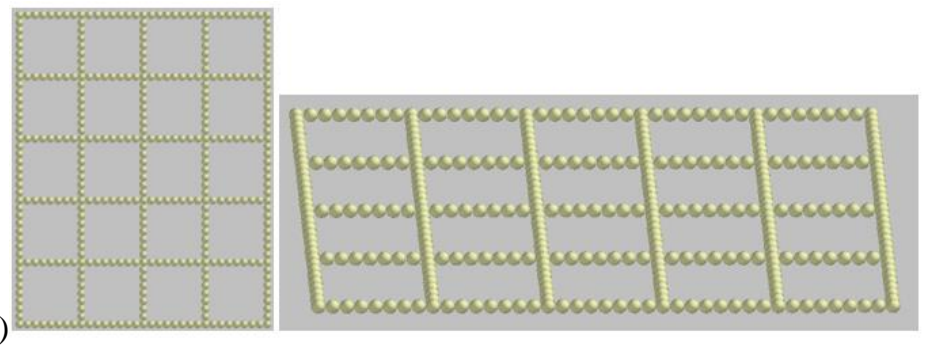

b)

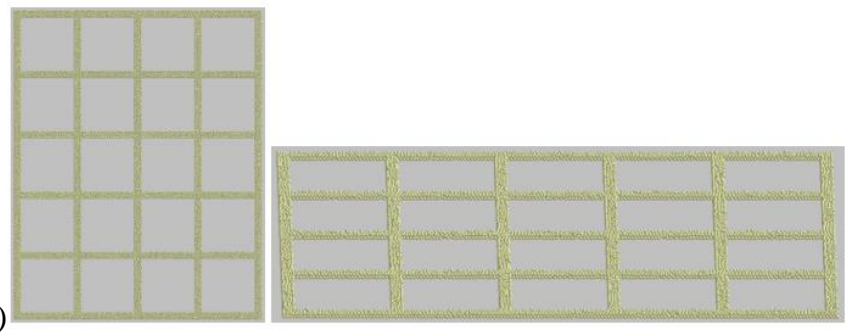

Fig. 4. General view of geogrid models: a) - model 1 with particle radius $R_{P}^{1}=0.002475 \mathrm{~m}$;b) - model 2, with particle radius $R_{\mathrm{P}}^{2}=0.000625 \mathrm{~m}$. 
Table 2. Parameters of geogrid models.Table 2 provides parameters accepted for simulation of both geogrids.

Table 2. Parameters of geogrid models.

\begin{tabular}{|l|c|c|c|}
\hline \multirow{2}{*}{ Parameters } & \multirow{2}{*}{ Units } & \multicolumn{2}{|c|}{ Values } \\
\cline { 3 - 4 } & $\mathrm{m}$ & Model 1 & Model 2 \\
\hline Radius of the bond & $\mathrm{N} / \mathrm{m} 3$ & 23529600000 & 2341200000000 \\
\hline Normal stiffness (strength) & $\mathrm{N} / \mathrm{m} 3$ & 23529600000 & 2341200000000 \\
\hline Shear stiffness & $\mathrm{N} / \mathrm{m} 2$ & 169723000 & $2.44462 \mathrm{e}+20$ \\
\hline Maximum normal stress & $\mathrm{N} / \mathrm{m} 2$ & 169723000 & $2.44462 \mathrm{e}+20$ \\
\hline Maximum shear stress & $\mathrm{m}$ & 0.002475 & 0.000625 \\
\hline Radius of particles &
\end{tabular}

Since the tests were carried out to determine the stress at $2 \%$ and $5 \%$ tension of the materials, i.e. the geogrid models were not brought to destruction, then the values of the maximum stresses that can occur in the bonds between the particles are assumed to be overestimated. It should be noted that the second model of the geogrid used particles not aligned in the same plane as the first one, which led to the appearance of bonds not only in the direction of the material tension (elongation), but perpendicular to it. Therefore, the value of maximum stresses is even more overestimated in order to exclude single local breakage of the bonds between the particles. Otherwise, this could lead to a sharp increase in broken bonds and the destruction of the sample as a whole. In other words, the possible defect or preliminary partial damage of the material is not taken into account, for example, during transportation and laying out on the site.

\section{Test simulation}

There is a document [7] in the Russian Federation that regulates the testing of geogrids for determination of their relative elongation under tensile force. In accordance with the requirements of this document, the tension (elongation) parameter is determined by function (2), and the tension force by function (3)

$$
\varepsilon_{2,5 \%}=\frac{\Delta 1}{1_{0}} \times 100 \%,
$$

where $\Delta \mathrm{l}$ - length increase of the sample, $\mathrm{mm} ; \mathrm{l}_{0}$ - initial length, $\mathrm{mm}$.

$$
\mathrm{F}_{2,5 \%}=\frac{\mathrm{F} \times \mathrm{N}_{\mathrm{t}}}{\mathrm{N}_{\mathrm{r}}},
$$

where $\mathrm{F}$ - maximum force, mm; $\mathrm{N}_{\mathrm{t}}$ - number of elements (edges) per unit width, pcs; $\mathrm{N}_{\mathrm{r}}$ - number of elements (edges) in cross-section, pcs.

In accordance with the requirements of [7], the speed of clamp movement at the moment of testing should be $10 \mathrm{~mm} / \mathrm{min}$. It should be noted here that although this value was sustained in the course of computer modelling, it does not influence on the behavior of the material or the results of the test, if the reading periods are correctly established during the simulation process. 
In the frame of this work we developed special clamps for geogrids excluding slippage of the material (Fig. 5). Similar construction allows improving the stability of geogrid models in the process of testing.

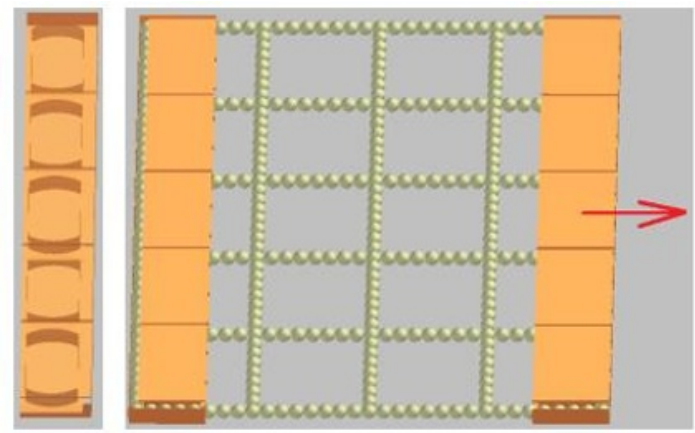

Fig. 5. General view of the clamp model.

To reduce the effect on the test results for "pressing" the geogrid particles into the clamps, the deformation characteristics of these materials must be different. Those parameters for clamps were almost 10 times higher. It should be noted that sharp speed-up of the clamp should not be allowed during the test, as this can lead to inaccuracies in the data obtained during the test.

\section{Test results}

The process of computer simulation allows you to determine stresses that occur not only in the clamps, but also in parts of the geogrid. This is noted in articles [5,6]. Figure 6 graphically shows the distribution of stresses in the edges of geogrids.
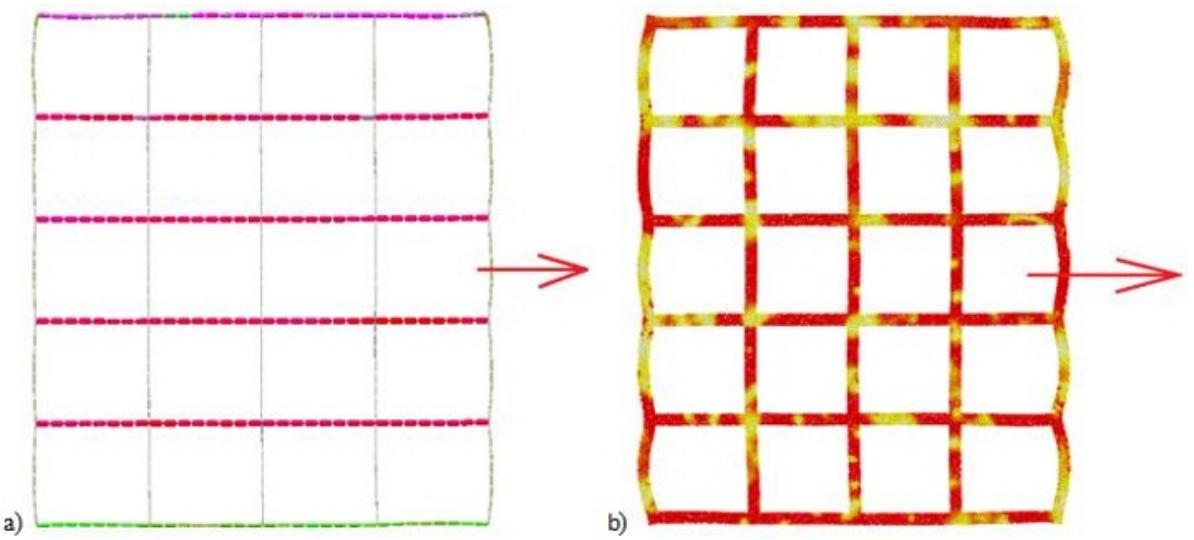

Fig. 6. General view of stress distribution between particles: a) - model 1; b) - model 2 .

The obtained information during problem solution No. 3, indicated in the introduction of this article, will make it possible to determine the nature of the interaction between the soil particles and geosynthetic materials more effectively and, as a consequence, evaluate the correctness of parameter selection for reinforcing materials. Based on the results of the tests performed, we obtained curves of the material tension (elongation) to applied stresses ratio (Figure 7). It should be noted that in the process of these studies, the Hertz-Mindlin model did not allow to obtain the values of the tensile force corresponding to the values of the natural sample. Perhaps this is due to the nonlinear "stress-tension" ratio of the behavior of 
natural geogrid patterns, either due to the method of performing these tests, and possibly due to the combination of computer model parameters. In any way, the research data will follow the parameters presented in this article.

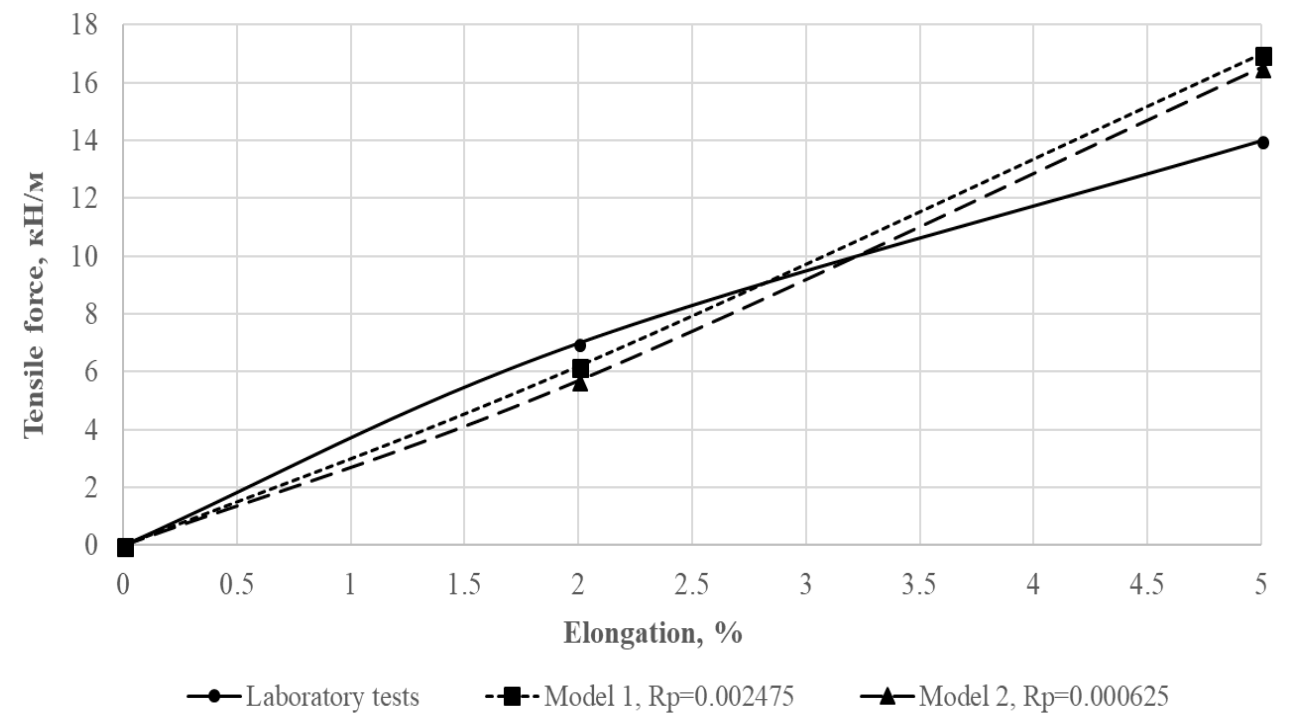

Fig. 7. Curves of material elongation (tension) to tensile force (or stress) ratio.

Based on the obtained results we can make the following conclusions:

1. Parameters of geogrid models allow accurately defining the behavior of a natural sample when performing laboratory tests in determining the stresses causing an elongation of $2 \%$ and $5 \%$ [7];

2. The used Hertz-Mindlin model allows to accurately solve the problem of computer simulation of a geogrid corresponding to the characteristics of a natural reinforcing material within the framework of solving problems that do not imply bringing the reinforced structure to destruction;

3. Model 2 showed great stability in testing, but it requires more efforts to create a uniform material structure, in comparison to model 1 ;

4. The clamp structure plays an important role. The variant presented in the article allows to minimize the influence of the effect of "pulling" the material from the clamp on the test results.

\section{Conclusion}

We obtained two models of geogrids complying with the real material. Thus, using DEM we solved problem №1, as well as obtained the method for the formation of material structure and its testing. Further studies will allow us to get computer models of geosynthetic materials with various parameters.

To simplify the work of researchers it is necessary to create, for example, a nomogram that makes it possible to determine the parameters of a geogrid model with specific strength and deformation characteristics. This requires obtaining more computer models to determine the dependencies of model characteristics on its behavior under stresses during the test.

\section{References}


1. B. Radovsky, Road engineering II, 24-43 (2017)

2. A. Moshenzhal, Advanced design of road pavements with constructive layers from loosely bound soils, reinforced by geosynthetic materials, diss. candidate of engineering sciences (FSBEI Pacific National University, Khabarovsk, 2017)

3. https://www.edemsimulation.com/edem-2018-1-documentation/

4. G. Brau, Recommendations for design and analysis of Earth structures using geosynthetic reinforcements (EBGEO, Germany, 2011)

5. T. Ngoc, A study of the geogrid-subballast interface via experimental evaluation and discrete element modelling, Granular Matter (2017)

6. T. Ngoc, Modelling geogrid-reinforced railway ballast using the discrete element method, Transportation, Geotechnics (2016)

7. ODM 218.5.002-2008 Methodical recommendations for the use of polymeric geogrids for reinforcing pavement layers from granular materials: approved by the Federal Road Transport Agency 